\title{
A Measurement of Non-Coaxial RF Devices with Improved TRL Calibration Algorithm
}

\author{
Chen Shouhong ${ }^{1}$, Wang Zhuang ${ }^{1}$, Ma Jun ${ }^{1, *}$, and Hou Xingna ${ }^{2}$ \\ ${ }^{1}$ School of Electronic Engineering\&Automation, Guangxi Key Laboratory of Automatic Detecting Technology and Instruments, Guilin \\ University of Electronic Technology Guilin, 541004, China \\ ${ }^{2}$ Guilin University of Electronic Technology Guilin, 541004, China
}

\begin{abstract}
In order to test the S-parameters of non-coaxial RF (Radio Frequency) devices accurately, using ADS (Advanced Design System) to simulate and design a set of fixture and calibration pieces for noncoaxial RF device performance test, and the calibration pieces is used to calibrate the test fixture, remove the error introduced by the fixture, and obtain the real $\mathrm{S}$ parameters of non-coaxial RF microwave devices. Based on the study of various parameters of RF microwave devices, the Engen's TRL (Thru-Reflect-Line) calibration algorithm is studied in depth. An improved TRL calibration algorithm is proposed. And the use of MATLAB program to achieve two calibration algorithms to correct the system error introduced by the measuring fixture, so as to get the real performance parameters of the device under test. Through the test of the verification device, the agreement between the improved TRL algorithm and Engen's TRL algorithm is verified. The final design of the test fixture can be successfully applied to the network analyzer for noncoaxial RF devices S-parameter measurement.
\end{abstract}

\section{Introduction}

RF (Radio Frequency) devices are widely used in military, research and civil fields, such as communications and navigation, electronic warfare, millimeter wave imaging, etc. According to different functions, RF devices mainly include amplifiers, filters, mixers and couplers. The main parameters include: Sparameter, $\mathrm{P} 1 \mathrm{~dB}$, frequency range, NF (Noise Figure), phase noise and so on. These performance parameters are the basis for RF device applications and evaluation, so the method used for parameter measurement is especially important. S parameters are important technical parameters of RF devices.

At present, the S-parameter test equipment for RF devices is network analyzer, the test connection port of the network analyzer is coaxial interface generally, and the input / output ports of the RF device are not coaxial generally. Therefore, when testing the S parameters of a $\mathrm{RF}$ device, a conversion circuit or test fixture needs to be provided between the coaxial network interface of the vector network analyzer and the RF device under test, the test fixture converts the non-coaxial RF interface to a coaxial interface that matches the network analyzer. As the fixture will affect the RF test results of the device, therefore, it is necessary to set the test end face to the RF device pin, that is, the calibration end surface extends from the end of the coaxial connector on the test fixture to the pin of the RF device under test. Then the error introduced in the test fixture is analyzed, according to the analysis results, those who can reduce the error factor, to further optimize the fixture circuit, those who cannot further reduce the system error to achieve the best compensation.

Compared with DC and low-frequency devices, the working wavelength of RF devices is very short, and the small physical size of the test fixture can also have a great impact on the device parameter test results. Therefore, need precision, scientific design test fixtures, and use the calibration fixture on the test fixture by means of a certain algorithm for de-embedding. The calibrated fixture can be used to test RF devices. At the same time, a series of calibration methods are used to accurately measure the performance of the conversion fixture to compensate for the influence of actual test results. Calibration is usually done using the TRL algorithm, calibrate with Thru, Reflect and Line.

In recent years, the TRL algorithm has proven to be useful in wafer devices and microwave circuit testing1. In the algorithm of TRL, the error model is ultimately attributed to the determination of an error, including the symbolic decision on the root of the error. Y. Liu et al. (2010) put forward a new method to solve the error term, the eight error terms caused by the fixture and the two leakage errors are derived directly from the S-parameters of the calibration standard measured from the coaxial reference plane without converting the S-parameters to T-parameters2. Rolain et al. (2015) studied an alternative is proposed for the line pairing process that underspans the M-TRL calibration3. Kuhlmann (2016). the effect of the standard characteristic impedance on the quality of the TRL calibration is described4.

\footnotetext{
* Corresponding author: majun@guet.edu.cn
} 


\section{Network nanlyzer callbration theory}

SOLT(Short-Open-Load-Thru) calibration method is a common calibration method5. Short, Open, Load, Through, four calibration pieces represent the four extreme cases, the accuracy of the calibration is based on the exact parameters of each calibration. Implementing SOLT calibrations in applications such as fixture testing, wafer testing, etc. is impractical or does not give the precise parameters of these calibrations. TRL calibration can overcome the shortcomings of the SOLT calibration, TRL Calibration Method Through the three calibration pieces of Through, Reflect, Line to realize the calibration of network analyzer, this method of calibration reduces the dependence on the exact parameters of the calibration part during the calibration process. Depending on how the calibration pieces is used, calibration methods such as LRM and LRL can also be used. For non-coaxial devices under test, such as waveguides and wafers, the TRL method is often used to calibrate, to overcome the non-coaxial and high frequency conditions of the test accuracy is not allowed. The complete TRL calibration determines the eight error models as shown in Figure. 1.

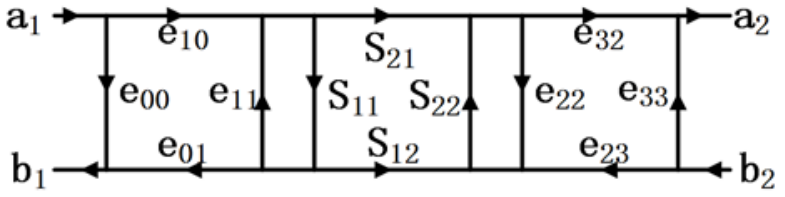

Figure. 1. Error model signal flow diagram

The TRL algorithm includes three calibration models6, Thru, Reflect, Line respectively represent the three opposite extreme cases, The transmission of the signal in the three calibration parts is described in Figure. 2, RA and RB sub-table shows the error introduced by the fixture at both ends of the device under test, and $\mathrm{RT}=\mathrm{RARB}, \mathrm{RD}=\mathrm{RARLRB}, \mathrm{By}$ connecting Thru (S11 $=\mathrm{S} 22=0, \quad \mathrm{~S} 12=\mathrm{S} 21=1), \quad$ Reflect $(\mathrm{S} 11=\mathrm{S} 22=\Gamma$ $\mathrm{R})$ and Line $(\mathrm{S} 11=\mathrm{S} 22=0, \mathrm{~S} 12=\mathrm{S} 21=\mathrm{e}-\gamma \mathrm{l})$ to the test network for three tests, And obtain three sets of $S$ parameters from the coaxial reference plane. Each group contains four S parameters: S11, S21, S12 and S22.

The purpose of using TRL calibration parts is to utilize the extreme RF characteristics of the calibration parts to solve the system of equations to find the eight errors and eliminate the error term. Then the actual $\mathrm{S}$ parameters of the RF microwave device to be measured are deduced according to the system flow chart. By solving the equations, you get the true S-parameter equation of the device under test, which includes the eight errors introduced by the fixture, resulting in equation 错误!未找到引用源。).

$$
R=\frac{1}{r_{22} \rho_{22}} \frac{1}{a \alpha} \frac{1}{1-b \frac{c}{a}} \frac{1}{1-\gamma \frac{\beta}{\alpha}}\left[\begin{array}{cc}
1 & -b \\
-c & a
\end{array}\right] R_{M}\left[\begin{array}{cc}
1 & -\beta \\
-\gamma & \alpha
\end{array}\right]
$$

Although there are eight errors, r22 $\rho 22$ can be combined into one error after resolution, calculating the values of $a, b, c, \alpha, \beta, \gamma$ and $r 22 \rho 22$ can correct the systematic errors introduced during the test and get the true $\mathrm{S}$ parameters of the device under test.

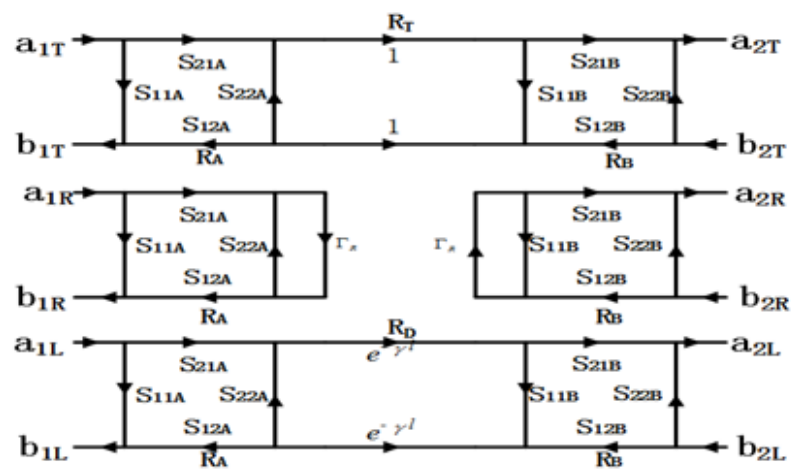

Figure. 2. TRL connection model

\section{Simulation callbration pieces}

Three calibrators of Thru, Reflect and Line were simulated by ADS software. The Rogers RO4350 was used as the substrate. The dielectric constant of the substrate was 3.66. The thickness of the plate was $0.762 \mathrm{~mm}$ and the thickness of the copper plate was $70 \mathrm{um}$. Find the optimal line width through simulation to achieve a good impedance matching, the final line width is set to $1.442 \mathrm{~mm}$.

\subsection{Thru Pieces}

When the electrical length of the pass-through calibration part is 0 , there is no loss and no reflection, and the transmission coefficient is 1 , that is $\mathrm{S} 12=\mathrm{S} 21=$ $1(0 \mathrm{~dB})$, When the electrical length is greater than 0 , the characteristic impedance of the pass-through calibration part must be the same as the characteristic impedance of the transmission calibration part, and does not need to measure insertion loss 6 . When the operating frequency is too high, the $1 / 4$ wavelength delay line has a short physical size and is poorly fabricated. In this case, the delay line is increased in size to increase its physical size. We set the length of the pass-through calibration part to $28.78 \mathrm{~mm}$, as shown in

Fig. 3.

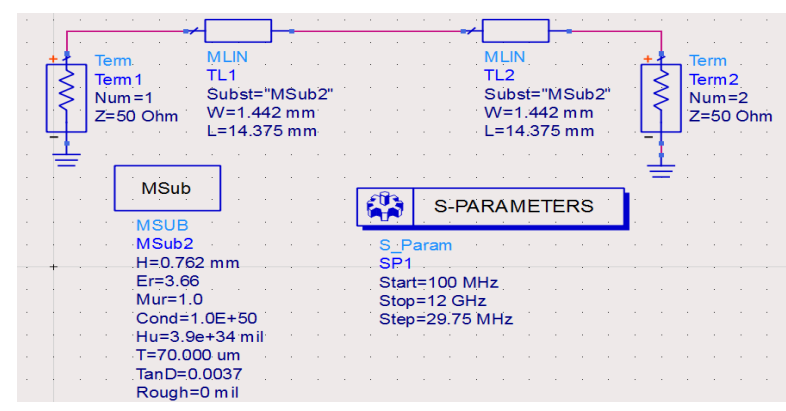

Fig. 3. Through schematic

In the simulation results, $\mathrm{S} 11$ of pass-through calibration unit is lower than $-20 \mathrm{~dB}$ in $0 \sim 12.5 \mathrm{G}$ range, 
$\mathrm{S} 21$ is greater than $-0.183 \mathrm{~dB}$, the simulation results are good, as shown in Fig. 4.
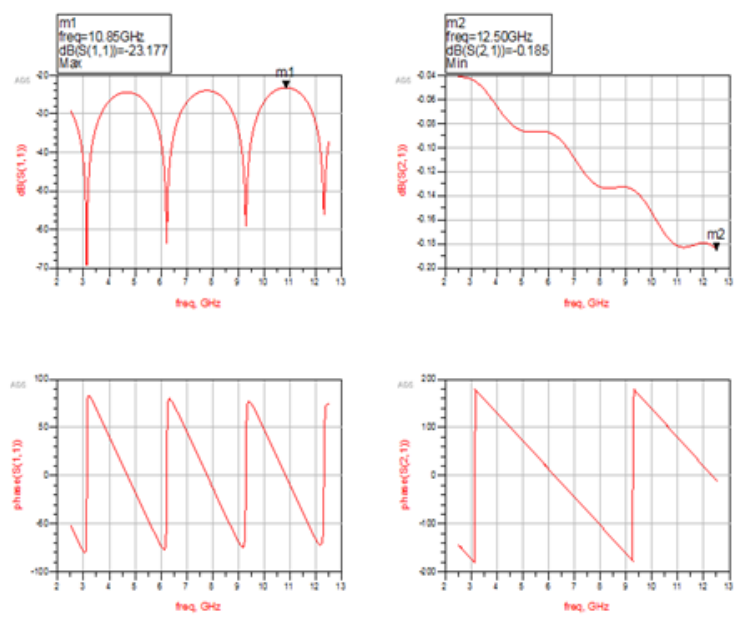

Fig. 4. Through Results

\subsection{Reflection Pieces}

Reflectance calibration of all ports must have the same reflection coefficient, reflection coefficient cannot know, but should be close to 1, Phase to ensure that the positive and negative 90 degrees or less 7 , that is, the reflection calibration of the signal in the transmission process almost no transmission, most of the signal is reflected back. The schematic diagram using ADS simulation reflection calibration is shown in

Fig. 5.

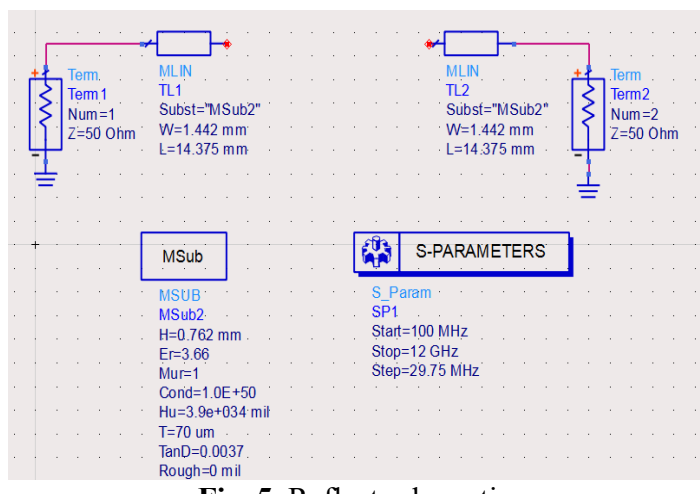

Fig. 5. Reflect schematic

In the simulation results, the S11 of the reflection pieces element is greater than $-0.16 \mathrm{~dB}$ in the range of $0 \sim 12.5 \mathrm{G}, \mathrm{S} 21$ is negative infinity, and the simulation result is good, as shown in Fig. 6.
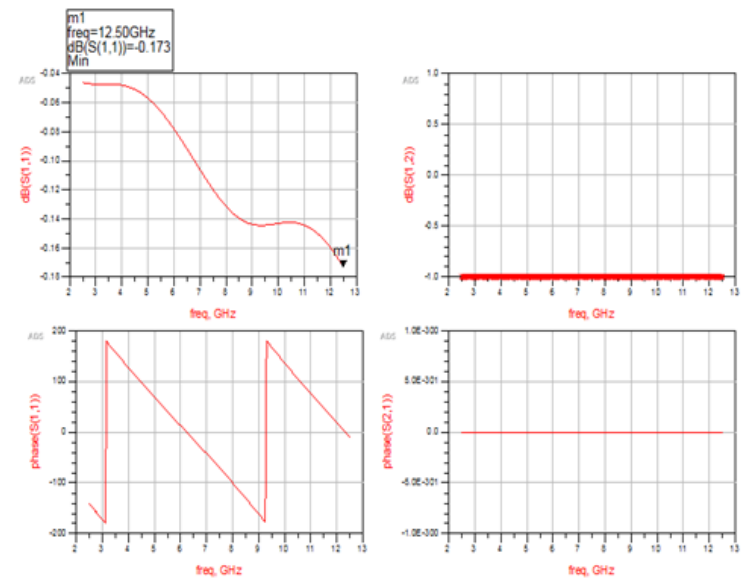

Fig. 6. Reflect results

\subsection{Line Pieces}

The transmission line connecting port 1 and port 2 does not need to know the length of the line. In general, the value of the phase difference is generally 90 degrees or the wavelength of the test signal is different according to needs, and the $1 / 4$ wavelength is taken as the value of the phase difference of the transmission line8. In the meantime, if the frequency of the signal to be measured is too high, the physical size of the $1 / 4$ wavelength transmission line will become very short, which may be very difficult to manufacture. In this case, a non-zero length pass-through calibration should be selected as the transmission line, using the required phase difference to determine the size of the transmission line9. When the measured frequency of the device operating frequency span and the initial frequency ratio greater than 8 , you should use more than one extension line, to ensure coverage of the entire frequency range. $2.5 \mathrm{GHz} \sim$ $12.5 \mathrm{GHz}$ delay line simulation schematic diagram is as shown in Fig. 7.

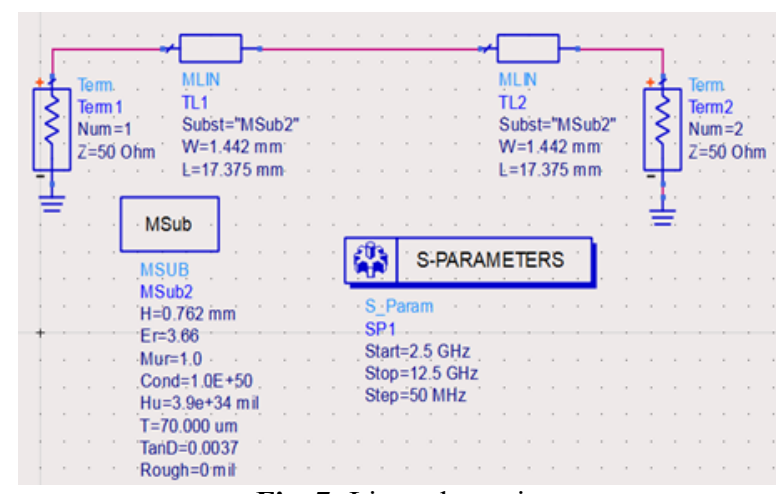

Fig. 7. Line schematic

We designed a $2.5 \mathrm{Ghz}$ to $12.5 \mathrm{Ghz}$ delay line, $12.5 \mathrm{Ghz} 1 / 4$ wavelength of $6 \mathrm{~mm}$, the length of the delay line $6 \mathrm{~mm}$ longer than the straight piece.S11 are less than $-23.008 \mathrm{~dB}, \mathrm{~S} 21$ are greater than $-0.229 \mathrm{~dB}$, simulation result is shown in Fig. 8. 

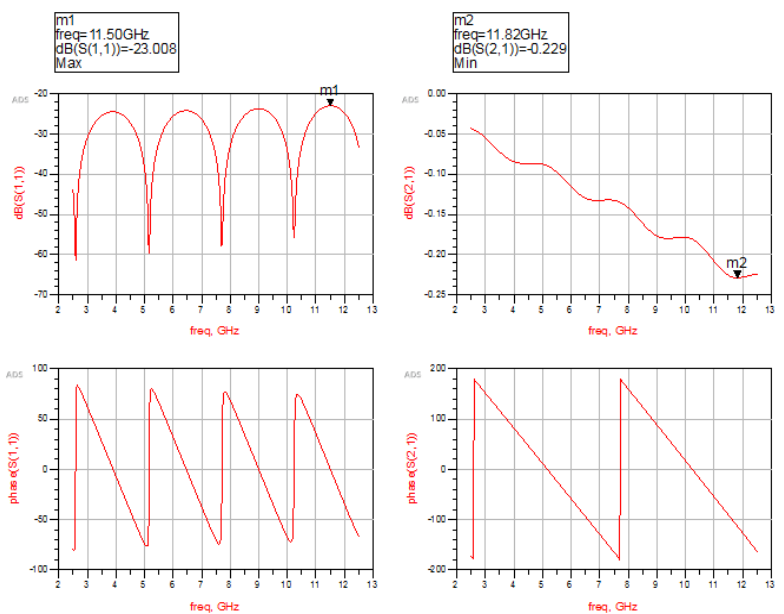

Fig. 8. Line results

\section{Test results}

We tested Murata's GRM1555C2A1R0CA01 capacitor, and we downloaded Library for Keysight ADS at Murata's official website. We imported this model in ADS and tested it as shown in Fig. 9.

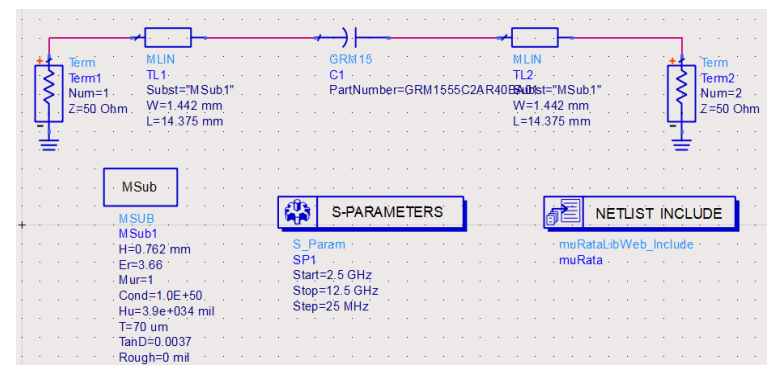

Fig. 9. DUT schematic

Test simulation results as shown in Fig. 10.
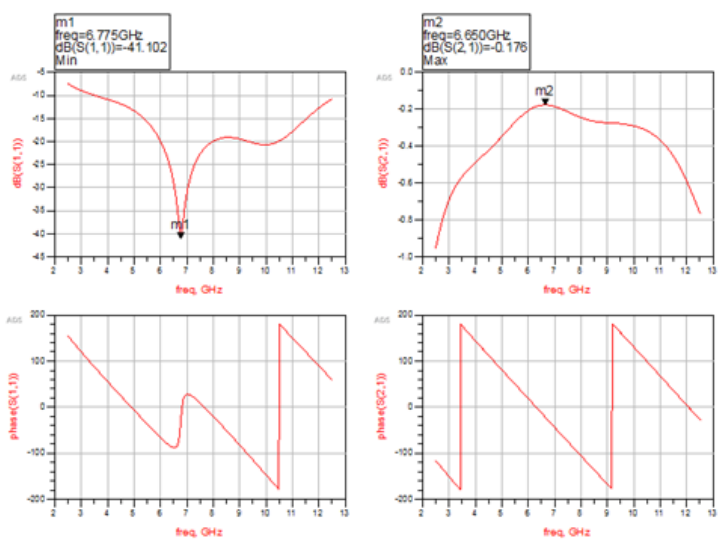

Fig. 10. DUT results

According to the Network Analyzer Calibration Theory introduced in Section 2, the test data is calibrated using MATLAB, and the calibration result is as shown in Fig. 11.

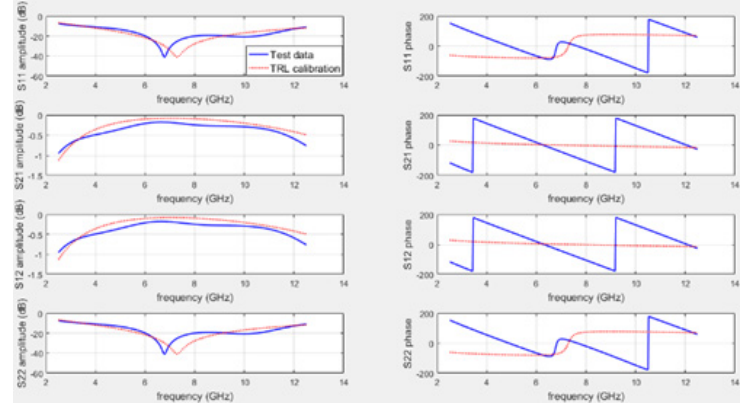

Fig. 11 TRL calibration result

Comparison of calibration results and Murata official website of the data, It can be seen that the calibrated data has good consistency with the Murata website data, the comparison results as shown in Fig. 12.
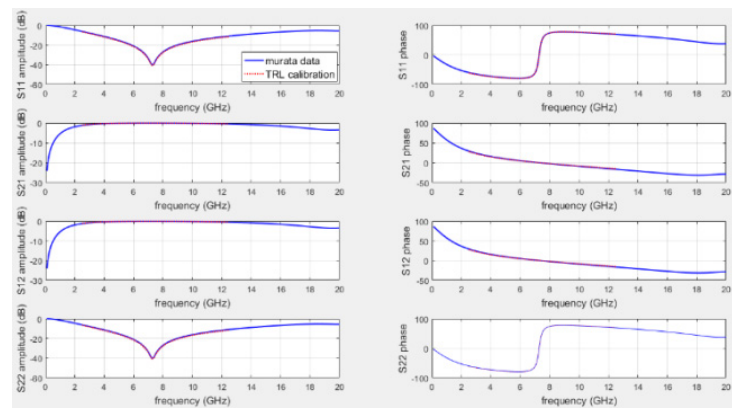

Fig. 12. Data comparison

\section{Conclusions}

In this paper, we designed and optimized the non-coaxial $\mathrm{RF}$ microwave test fixtures using ADS based on RF microwave transmission line theory. We designed a set of S-parameter test fixtures and fixtures calibrators for non-coaxial RF devices, and use the improved TRL Embedding algorithms for de-embedding calibration of test fixtures. The results shows that the calibration results are good, suitable for RF fixture calibration. The test fixture and algorithm used in the testing of noncoaxial RF devices can stably calculate and calibrate the test data of non-coaxial RF devices, providing great convenience for the testing of RF devices.

\section{Acknowledgements}

The authors would like to acknowledge the support of Natural Science Foundation of Guangxi Province (No. 2015GXNSFDA139003); Guangxi Key Laboratory of Automatic Detecting Technology and Instruments (No. YQ14115, YQ15101).

\section{References}

1. Engen, G. F. and Hoer, C. A. Thru-Reflect-Line: An Improved Technique for Calibrating the Dual Six-Port Automatic Network Analyzer. IEEE 
Transactions on Microwave Theory \& Techniques, 27(12), 987-993(1979)

2. Liu, Y., Tong, L., Tian, Y., and Gao, B. Measurements of planar microwave circuits using an improved trl calibration method. Progress in Electromagnetics Research, 109(4), 263-278(2010)

3. Rolain, Y., Ishteva, M., Nechel, E. V., and Ferranti, F. A tensor-based extension for the multi-line trl calibration. IEEE Transactions on Microwave Theory \& Techniques, 64(7), 2121-2128(2016)

4. Kuhlmann, K. A study of the reference impedance influence on a TRL calibration. Microwave Measurement Conference (pp.1-4). IEEE(2016)

5. Wang, M., Zhao, Y. J., Jin, Y. M., and Zhou, Y. G. Sensitivity analysis of multiport s-parameter measurements due to nonideal trl calibration standards. Radio Science(2017)

6. Lu, H., Zhou, Z., Chengwei, Zhou, J., Chen, T., and Chen, C. A new on-wafer multiline thru-reflect-line (TRL) calibration standard design. Antennas and Propagation (pp.934-936). IEEE(2014)

7. Wang, Y. B., Luan, P., Wu, A. H., Liang, F. G., and Sun, J. An accurate determination method of characteristic impedance of transmission line based on multi-trl algorithms. Jiliang Xuebao/acta Metrologica Sinica, 38(2), 225-229(2017)

8. Liu, S., Ocket, I., Lewandowski, A., Schreurs, D., and Nauwelaers, B. An improved line-reflectreflect-match calibration with an enhanced load model. IEEE Microwave \& Wireless Components Letters, 27(1), 97-99(2017)

9. Ye, X., Fan, J., Chen, B., and Drewniak, J. L. Accurate characterization of PCB transmission lines for high speed interconnect. Electromagnetic Compatibility (pp.16-19). IEEE(2015) 